\title{
Ingtegral equations with delaying arguments for semi-Markovian processes
}

\author{
Selahattin Maden ${ }^{1}$, Ulviyya Y. Karimova ${ }^{2}$ and Tamilla I. Nasirova ${ }^{3}$ \\ ${ }^{1}$ Department of Mathematics, Faculty of Arts and Sciences, Ordu University, Ordu, Turkey \\ ${ }^{2}$ Department of International Relations and Economy, Baku State University, Baku, Azerbaijan \\ ${ }^{3}$ Department of Applied Mathematics and Cybernetics, Baku State University, Baku, Azerbaijan
}

Received: 5 January 2017, Accepted: 19 May 2017

Published online: 25 August 2017.

\begin{abstract}
In this paper, the Laplace transform of the distribution of the duration of a particular semi-Markovian random walk period is obtained in the form of the difference equation.
\end{abstract}

Keywords: Laplace transforms, semi-Markovian random process, random variable, process with positive tendency and negative jumps.

\section{Introduction}

In recent years, random walks with one or two barriers are being used to solve a number of very interesting problems in the fields of inventory, queues and reliability theories, matematical biology etc. Many good monographs in this field exist in literature (see [1], [3] and etc.). In particular, a number of very interesting problems of stock control, queues and reliability theories can be expressed by means of random walks with two barriers. These barriers can be reflecting, delaying, absorbing, elastic, etc., depending on concrete problems at hand. For instance, it is possible to express random levels of stock in a warehouse with finite volumes or queueing systems with finite waiting time or sojourn time by means of random walks with two delaying barriers. Furthermore, the functioning of stochastics systems with spare equipment can be given by random walks with two barriers, one of them is delaying and the other one is any type barrier.

It is known that the most of the problems of stock control theory is often given by means of random walks or random walks with delaying barriers (see [1-3] and etc.). Numerious studies have been done about step processes of semi-Markovian random walk with one or two barriers of their practical and theoretical importance. But in the most of these studies the distribution of the process has free distribution. Therefore the obtained results in this case are cumbersome and they will not be useful for applications (see [1-4] and etc.).

A number of studies have been carried out on the examination of the duration of a given half-Markov random walk in any interval. Busarov [2] is considered the asymptotic behavior of random wanderings in random medium with delaying screen. Lotov $[5,6]$ is studied random walks wandering in a stripe. The asymptotic of distributions in two-sided boundary problems for random walks defined on a Markov chain is given in [3]. Nasirova [7,8] is studied the various semi-markov 
random walk processeswith or without barriers. The Laplace transform for the distribution of the lower boundary functional in a semi-Markovian random walk with delaying barrier at zerolevel in [5]. Nasirova and Omarova [9] are given the Laplace transform of the time of the first hit of a delaying screen at zero was determined for the process of semi-Markov random walk with positive drift and negative jumps.

\section{Statement of the problem}

Let a sequence of independent and identically distributed pairs of random variables $\left\{\xi_{k}^{+}, \eta_{k}^{+}\right\}, k=\overline{1, \infty}$ and $\left\{\xi_{k}^{-}, \eta_{k}^{-}\right\}$, $k=\overline{1, \infty}$, defined on a probability space $(\Omega, F, P) \operatorname{such}$ that $\xi_{k}^{ \pm}$and $\eta_{k}^{ \pm}$are independent random variables and $\xi_{k}^{ \pm}>0, \eta_{k}^{ \pm}>$ 0 . Using these random variables we will derive the following step processes of semi-Markovian random walk.

$$
\begin{aligned}
X^{+}(t) & =\sum_{i=1}^{k-1} \eta_{i}^{+}, \text {if } \sum_{i=1}^{k-1} \xi_{i}^{+} \leq t<\sum_{i=1}^{k} \xi_{i}^{+} \\
X^{-}(t) & =\sum_{i=1}^{k-1} \eta_{i}^{-}, \text {if } \sum_{i=1}^{k-1} \xi_{i}^{-} \leq t<\sum_{i=1}^{k} \xi_{i}^{-}
\end{aligned}
$$

Also let us defined the following semi-Markovian process.

$$
X(t)=X^{+}(t)-X^{-}(t)
$$

A view of this semi-Markovian random walk process is as follows.

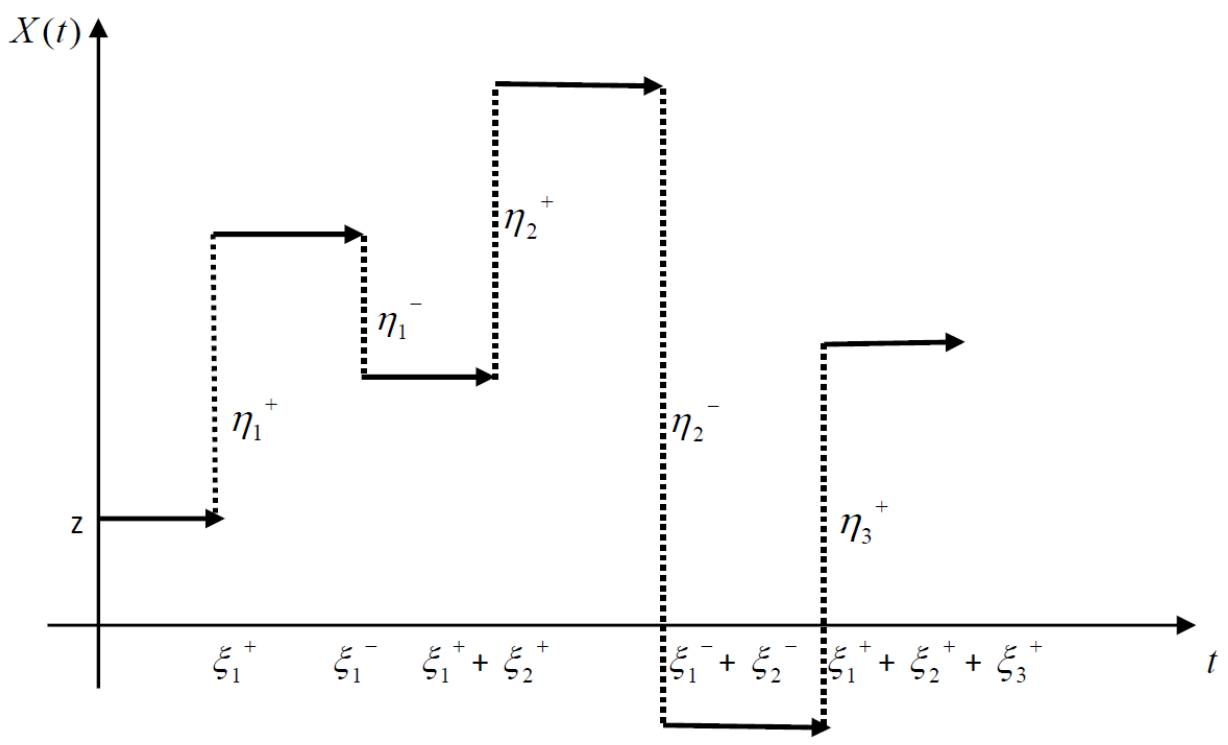

The main aim of this study is to express the Laplace transform of the distribution function for the duration of the process $\mathrm{X}(t)$ by means of some probability characteristics of random variables $\xi_{k}^{ \pm}$and $\eta_{k}^{ \pm}$. 
Assume that the random variable $\xi_{1}^{+}$has an exponential distribution. Therefore the process $X(t)$ is reduce to any complex markov prosess. Also let's the process $\mathrm{X}(t)$ is stay in any interval $[a, b]$, where $a>0, b>0$.

We introduce the foolwing notations.

$\tilde{L}(\theta ; x)=\int_{t=0}^{\infty} e^{-\theta t} L(t ; x) d t$ - the Laplace transform of the function $L(t ; x)$ with respect to $t$.

$$
\begin{aligned}
& \mathrm{A}_{z}=\left\{\inf _{0 \leq s \leq t} X(s)>b ; \sup _{0 \leq s \leq t} X(s)<a \mid X(0)=z\right\}, z<0 \\
& K(t ; b, a \mid X(0)=z)=P\left\{\inf _{0 \leq s \leq t} X(s)>b ; \sup _{0 \leq s \leq t} X(s)<a \mid X(0)=z\right\} \\
& \tilde{K}(\theta ; b, a \mid X(0)=z)=\int_{t=0}^{\infty} e^{-\theta t} P\left\{\inf _{0 \leq s \leq t} X(s)>b ; \sup _{0 \leq s \leq t} X(s)<a \mid X(0)=z\right\} d t
\end{aligned}
$$

which is obtained from (2) by multiplying both of side this equation with $e^{-\theta t}, \theta>0, t>0$ and then integrating from zero to $\infty$ with respect to $t$.

Therefore the event $A_{z}$ occur one of them the following cases.

$$
\begin{aligned}
& H_{1}=\left\{\xi_{1}^{+}>t ; \xi_{1}^{-}>t\right\}, H_{2}=\left\{\xi_{1}^{+}<\xi_{1}^{-}<t\right\}, \\
& H_{3}=\left\{\xi_{1}^{+}<t<\xi_{1}^{-}\right\}, H_{4}=\left\{\xi_{1}^{-}<\xi_{1}^{+}<t\right\}, \\
& H_{5}=\left\{\xi_{1}^{-}<t<\xi_{1}^{+}\right\} .
\end{aligned}
$$

On the other hand if we denote $K(t ; b, a \mid X(0)=z)=K(t \mid z)$, then we can write

$$
\begin{aligned}
K(t \mid z) & =P\left(A_{z} \bigcup_{i=1}^{5} \mathrm{H}_{i}\right)=\mathrm{P}\left(\mathrm{A}_{z} \mathrm{H}_{1}\right)+\mathrm{P}\left(\mathrm{A}_{z} \mathrm{H}_{2}\right)+\mathrm{P}\left(\mathrm{A}_{z} \mathrm{H}_{3}\right)+\mathrm{P}\left(\mathrm{A}_{z} \mathrm{H}_{4}\right)+\mathrm{P}\left(\mathrm{A}_{z} \mathrm{H}_{5}\right) \\
& =P\left\{\xi_{1}^{+}>t ; \xi_{1}^{-}>t \mid X(0)=z\right\} \\
& \int_{y=b}^{a} \int_{s=0}^{t} P\left\{\xi_{1}^{+}<s ; \xi_{1}^{-} \in d s ; X(s) \in d y \mid X(0)=z\right\} K(t-s ; \mid y) \\
& +\int_{y=b}^{a} \int_{s=0}^{t} P\left\{\xi_{1}^{+}<d s ; \xi_{1}^{-}>t ; X(s) \in d y \mid X(0)=z\right\} K(t-s ; \mid y) \\
& +\int_{y=b s=0}^{a} \int^{t} P\left\{\xi_{1}^{-}<s ; \xi_{1}^{+} \in d s ; X(s) \in d y \mid X(0)=z\right\} K(t-s ; \mid y) \\
& +\int_{y=b s=0}^{a} \int^{t} P\left\{\xi_{1}^{-} \in d s ; \xi_{1}^{+}>t ; X(s) \in d y \mid X(0)=z\right\} K(t-s ; \mid y) .
\end{aligned}
$$


From the independence of the random variables $\xi_{k}^{ \pm}$and $\eta_{k}^{ \pm}$, we have

$$
\begin{aligned}
K(t \mid z) & =P\left\{\xi_{1}^{+}>t ;\right\} P\left\{\xi_{1}^{-}>t\right\} \\
& +\int_{y=b}^{a} \int_{s=0}^{t} P\left\{\xi_{1}^{+}<s ; \xi_{1}^{-} \in d s ; X(s) \in d y \mid X(0)=z\right\} K(t-s \mid y) \\
& +P\left\{\xi_{1}^{-}>t\right\} \int_{y=b s=0}^{a} \int_{s=0}^{t} P\left\{\xi_{1}^{+}<d s ; X(s) \in d y \mid X(0)=z\right\} K(t-s \mid y) \\
& +\int_{y=b s=0}^{a} P\left\{\xi_{1}^{-}<s ; \xi_{1}^{+} \in d s ; X(s) \in d y \mid X(0)=z\right\} K(t-s \mid y) \\
& +P\left\{\xi_{1}^{+}>t ;\right\} \int_{y=b s=0}^{a} \int_{s}^{t} P\left\{\xi_{1}^{-} \in d s ; X(s) \in d y \mid X(0)=z\right\} K(t-s \mid y) .
\end{aligned}
$$

In this case, we can write

$$
\begin{aligned}
K(t \mid z) & =P\left\{\xi_{1}^{+}>t ;\right\} P\left\{\xi_{1}^{-}>t\right\} \\
& +\int_{y=b s=0}^{a} \int_{s}^{t} P\left\{\xi_{1}^{+}<s ; \xi_{1}^{-} \in d s ; \max \left[\min \left(a ; z+X^{+}(s)-\eta_{1}^{-} ; b\right] \in d y\right\} K(t-s ; \mid y)\right. \\
& +P\left\{\xi_{1}^{-}>t\right\} \int_{y=b}^{a} \int_{s=0}^{t} P\left\{\xi_{1}^{+} \in d s ; \min \left(a ; z+\eta_{1}^{+}\right) \in d y \mid\right\} K(t-s ; \mid y) \\
& \left.+\int_{y=b s=0}^{a} \int_{s}^{t} P\left\{\xi_{1}^{-}<s ; \xi_{1}^{+} \in d s ; \min \left[\max \left(b ; z-X^{-}(s)\right)+\eta_{1}^{+}\right), a\right] \in d y\right\} K(t-s ; \mid y) \\
& +P\left\{\xi_{1}^{+}>t\right\} \int_{y=b s=0}^{a} \int_{s}^{t} P\left\{\xi_{1}^{-} \in d s ; \max \left(b ; z-\eta_{1}^{-}\right) \in d y\right\} K(t-s ; \mid y)
\end{aligned}
$$

since $\xi_{1}^{+}>\xi_{1}^{-}$and $\xi_{1}^{+}<\xi_{1}^{-}$. Thus we have

$$
\begin{aligned}
K(t \mid z) & =P\left\{\xi_{1}^{+}>t ;\right\} P\left\{\xi_{1}^{-}>t\right\} \\
& +P\left\{\xi_{1}^{-}>t\right\} \int_{y=b}^{a} \int_{s=0}^{t} d_{y} P\left\{\xi_{1}^{+} \in d s ; \min \left(a ; z+\eta_{1}^{+}\right)<y\right\} K(t-s ; \mid y) \\
& \left.+\int_{y=b s=0}^{a} \int_{y}^{t} d_{y}\left\{\xi_{1}^{-}<s ; \xi_{1}^{+} \in d s ; \min \left[\max \left(b ; z-X^{-}(s)\right)+\eta_{1}^{+}\right), a\right]<y\right\} K(t-s ; \mid y) \\
& +P\left\{\xi_{1}^{+}>t ;\right\} \int_{y=b s=0}^{a} \int_{s}^{t} d_{y} P\left\{\xi_{1}^{-} \in d s ; \max \left(b ; z-\eta_{1}^{-}\right)<y\right\} K(t-s ; \mid y)
\end{aligned}
$$


by simplfying the equation (2). Since the random processes $\mathrm{X}^{+}(t)$ and $\mathrm{X}^{-}(t)$ are independent from the random variables $\xi_{1}^{+}$ve $\xi_{1}^{-}$respectivelly, according the the total probability formula, we can write

$$
\left.\left.P\left\{\xi_{1}^{-}<s\right\}=P\left\{\xi_{1}^{-}<s ; \min \left[\max \left(b ; z-X^{-}(s)\right)\right]+\eta_{1}^{+} ; a\right]<y\right\} \quad+P\left\{\xi_{1}^{-}<s ; \min \left[\max \left(b ; z-X^{-}(s)\right)\right]+\eta_{1}^{+} ; a\right]>y\right\} .
$$

Therefore the equation (2) is reduced to the following difference equation.

$$
\begin{aligned}
& \tilde{\mathrm{K}}(\theta / z)=\frac{1}{\lambda_{+}+\lambda_{-}+\theta}-\frac{\lambda_{+} \lambda_{-} \mu_{-}}{\left(\lambda_{+}+\theta\right) \theta} e^{-\mu_{-} a} \int_{y=b}^{a} \tilde{\mathrm{K}}(\theta \mid y) e^{\mu_{-} y} d y \\
& +\frac{\lambda_{+} \lambda_{-} \mu_{-}}{\left(\lambda_{+}+\theta\right)\left(\lambda_{-}+\theta\right)} e^{-\frac{\lambda_{+} \mu_{+}+\left(\mu_{+}+\mu_{-}\right) \theta}{\lambda_{-}+\theta}} e^{\frac{\mu_{+} \theta}{\lambda_{+}+\theta} z} \int_{y=b}^{a} \tilde{\mathrm{K}}(\theta \mid y) e^{\mu_{-} y} d y \\
& +\frac{\lambda_{+} \lambda_{-} \mu_{+} \mu_{-}}{\left(\lambda_{+}+\theta\right)\left(\lambda_{-}+\theta\right)} e^{\frac{\mu_{+} \theta}{\lambda_{+}+\theta} z} \int_{y=z}^{a} \tilde{\mathrm{K}}(\theta \mid y) e^{-\frac{\mu_{+} \theta}{\lambda_{+}+\theta} y} \int_{x=0}^{a-y} e^{-\frac{\left[\left(\lambda_{+}+\theta\right) \mu_{-}+\mu_{+} \theta\right]}{\lambda_{+}+\theta}} d x d y \\
& -\frac{\lambda_{+} \lambda_{-} \mu_{+} \mu_{-}}{\left(\lambda_{+}+\theta\right)\left(\lambda_{-}+\theta\right)} e^{\frac{\mu_{+} \theta}{\lambda_{+}+\theta} z} \int_{y=b}^{z} \tilde{\mathrm{K}}(\theta \mid y) e^{-\frac{\mu_{+} \theta}{\lambda_{+}+\theta} y} \int_{x=z-y}^{a-y} e^{-\frac{\left.\left[\lambda_{+}+\theta\right) \mu_{-}+\mu_{+} \theta\right]}{\lambda_{+}+\theta}} d x d y \\
& -\frac{\lambda_{+} \lambda_{-} \mu_{+}}{\left(\lambda_{-}+\theta\right) \theta} e^{\mu_{+} b} \int_{y=b}^{a} \tilde{\mathrm{K}}(\theta \mid y) e^{-\mu_{+} y} d y+\frac{\lambda_{+} \lambda_{-} \mu_{+}}{\left(\lambda_{+}+\theta\right)\left(\lambda_{-}+\theta\right)} e^{\frac{\lambda_{-} \mu_{+}+\left(\mu_{+}+\mu_{-}\right) \theta}{\lambda_{-}+\theta}} b e^{\frac{\mu_{-} \theta}{\lambda_{-}+\theta} z} \int_{y=b}^{a} \tilde{\mathrm{K}}(\theta \mid y) e^{-\mu_{+} y} d y \\
& +\frac{\lambda_{+} \lambda_{-} \mu_{+}}{\left(\lambda_{+}+\theta\right)\left(\lambda_{-}+\theta\right)} e^{\frac{\mu_{-} \theta}{\lambda_{-}+\theta} z} \int_{y=b}^{z} \tilde{\mathrm{K}}(\theta \mid y) e^{-\frac{\mu_{-} \theta}{\lambda_{-}+\theta} y} \int_{x=0}^{y-z} e^{-\frac{\left[\left(\lambda_{-}+\theta\right) \mu_{+}+\mu_{-} \theta\right]}{\lambda_{-}+\theta} x} d x d y \\
& +\frac{\lambda_{+} \lambda_{-} \mu_{+}}{\left(\lambda_{+}+\theta\right)\left(\lambda_{-}+\theta\right)} e^{\frac{\mu_{-} \theta}{\lambda_{-}+\theta} z} \int_{y=b}^{a} \tilde{\mathrm{K}}(\theta \mid y) e^{-\frac{\mu_{-} \theta}{\lambda_{-}+\theta} y} \int_{x=y-z}^{y-b} e^{-\frac{\left[\left(\lambda_{-}+\theta\right) \mu_{+}+\mu_{-} \theta\right]}{\lambda_{-}+\theta}} x d x d y \\
& +\frac{\lambda_{+} \mu_{+}}{\lambda_{+}+\lambda_{-}+\theta} e^{-\mu_{+} z} \int_{y=z}^{a} \tilde{\mathbf{K}}\left(\lambda_{-}+\theta \mid y\right) e^{-\mu_{+} y} d y+\frac{\lambda_{-} \mu_{-}}{\lambda_{+}+\lambda_{-}+\theta} e^{-\mu_{-} z} \int_{y=b}^{z} \tilde{\mathbf{K}}\left(\lambda_{+}+\theta \mid y\right) e^{\mu_{-} y} d y .
\end{aligned}
$$

\section{Competing interests}

The authors declare that they have no competing interests.

\section{Authors' contributions}

All authors have contributed to all parts of the article. All authors read and approved the final manuscript.

\section{References}

[1] Borovkov, A. A. (1976). Stochastic Processes in Queueing Theory, Springer Verlag, New York.

[2] Busarov, V. A. (2004). On asymptotic behaviour of random wanderings in random medium with delaying screen, Vest. Mos. Gos. Univ., 1(5), 61-63. 
[3] Feller, W. (1968). An Introduction to Probability Theory and Its Applications, Vol. I, Wiley, New York.

[4] Khaniev, T.A., Unver, I. (1997). The study of the level zero crossing time of a semi-Markovian random walk with delaying screen, Turkish J. Mathematics, 2(1), 257-268.

[5] Lotov, V. I. (1991a). On random walks in a band. Probability Theory and its Application, 36(1), 160-165.

[6] Lotov, V. I. (1991b). On the asymptotic of distributions in two-sided boundary problems for random walks defined on a markov chain, Sib. Adv. Math., 1(2), 26-51.

[7] Nasirova, . I. (1984). Processes of Semi-Markov Random Walk, ELM, Baku, 165p.

[8] Nasirova,. I., Ibayev, E. A., Aliyeva, T.A. (2005). The Laplace transformation of the distribution of the first moment reaching the positive delaying screen with the semi-Markovian process, Proc. Int. Conf. On Modern Problems and New Trends in Probability Theory, Chernivtsi, Ukraine, 19-26.

[9] Nasirova, I., Omarova, K. K. (2007). Distribution of the lower boundary functional of the step process of semi-Markov random walk with delaying screen at zero, Automatic Control and Computer Sciences, 59(7), 1010-1018.

[10] Omarova, K. K., Bakhshiev, Sh. B. (2010). The Laplace transform for the distribution of the lower bound functional in a semiMarkov walk process with a delay screen at zero, Automatic Control and Computer Sciences, 44(4), 246-252.

[11] Unver, I., Tundzh, Ya. S., Ibaev, E. (2014). Laplace-Stieltjes transform of distribution of the first moment of crossing the level $a(a>0)$ by a semi-Markovian random walk with positive drift and negative jmps, Automatic Control and Computer Sciences, 48(3), 144-149. 